\title{
Lupinenmehl - ein Allergen gewinnt an Bedeutung
}

\author{
Lupine Flour - An Allergen Gaining in Importance
}

Autoren

Institut
S. A. Grundmann, M. Mertens, S. Hungeling, R. Brehler

Abteilung für Allergologie, Klinik und Poliklinik für Hautkrankheiten, Universitätsklinikum Münster

\section{Bibliografie}

DOI $10.1055 / \mathrm{s}-2008-1077478$

Akt Dermatol 2008; 34 :

270-273 @ Georg Thieme

Verlag KG Stuttgart · New York ISSN 0340-2541

Korrespondenzadresse

Sonja A. Grundmann

Klinik und Poliklinik für

Hautkrankheiten

Universitätsklinikum Münster

Von-Esmarch-Str. 58

48149 Münster

Sonja.Grundmann@

ukmuenster.de

\section{Zusammenfassung \\ $\nabla$}

Eine 48-jährige Patientin stellte sich aufgrund oropharyngealer Symptome und Dyspnoe nach dem Genuss einer Rumkugel zur allergologischen Abklärung in unserer Ambulanz vor. Die initiale allergologische Diagnostik war bis auf den Nachweis einer Sensibilisierung gegenüber Soja unauffällig, diese stellte sich jedoch als klinisch nicht relevant heraus. Erst nach oraler Provokation mit der Rumkugel unter stationären Bedingungen und anschließender Herstelleranfrage

\section{Einleitung \\ $\nabla$}

Seitens des Verbrauchers und auch der Lebensmittelindustrie besteht wachsendes Interesse an alternativen pflanzlichen und zugleich preisgünstigen Proteinquellen - nicht zuletzt, weil die weltweit verbreitetste, das Soja, zunehmend im Ruf der genetischen Manipulation steht. Süßlupinensamen sind dem Soja hinsichtlich des Proteingehaltes ähnlich, weshalb Lupinen als das „neue Soja“ propagiert werden. Lupinen gehören wie Soja und Erdnüsse zur Familie der Leguminosen und werden von alters her zur Bodenverbesserung und wegen ihrer hohen biologischen Wertigkeit zur Verwendung als Tierfutter kultiviert. In der Ernährung des Menschen hatten Lupinen bisher nur eine geringe Bedeutung, vornehmlich als traditioneller Snack der Mittelmeerländer. Lupinenmehle und -eiweißkonzentrate sind seit Mitte der neunziger Jahre als Nahrungsmittelzusatz zugelassen und werden seither vermehrt, insbesondere glutenfreien Backwaren und Fertigprodukten, beigemengt. Sie zeichnen sich durch eine hohe Wasserbindungskapazität, gute emulgierende Eigenschaften und einen leicht nussigen Geschmack aus. Es wird behauptet, dass Lupine weniger allergen als Soja und deshalb besonders bezüglich der Zutaten konnte Lupinenmehl als auslösendes Allergen ausgemacht werden. Mittels Immunoblot-Inhibition konnte bei unserer Patientin eine primäre Sensibilisierung gegenüber Lupine nachgewiesen werden. Lupinenmehl wird zunehmend als Nahrungsmittelzutat, insbesondere in Backwaren, eingesetzt. Unser Fallbericht soll die Leserschaft für die wachsende Bedeutung dieses Allergens sensibilisieren und somit eine schnellere Diagnosefindung bei Lupinenallergien ermöglichen.

für die Ernährung von Milcheiweiß- und Soja-Allergikern geeignet sei. Allerdings hat parallel zur steigenden Verwendung dieser Hülsenfrucht die Zahl der berichteten IgE-vermittelten allergischen Reaktionen zugenommen.

\section{Kasuistik \\ $\nabla$ \\ Anamnese}

Eine 48-jährige Patientin berichtet bei der Vorstellung in unserer allergologischen Ambulanz, dass sie vierzehn Tage zuvor aufgrund einer allergischen Reaktion mit Niesen, Juckreiz, Halsschwellung, Atemnot und Kreislaufschwäche notärztlich versorgt werden musste. Sie habe eine Rumkugel aus einer im Supermarkt erworbenen Packung verzehrt und sei circa zehn Minuten später symptomatisch geworden. Ihr Ehemann habe sofort den Notarzt verständigt, sie sei noch zu Hause mit einer Kortison-Infusion behandelt und anschließend in die Notaufnahme des städtischen Krankenhauses gebracht worden. Die Symptome seien dort bereits rückläufig gewesen. Sie sei bis zum nächsten Morgen überwacht worden und ihr sei dringlich zu einer fachallergologischen Abklärung geraten worden. In 
der weiteren Anamnese ergab sich kein Hinweis für rhinokonjunktivale Beschwerden, Asthma bronchiale oder Nahrungsmittelunverträglichkeiten.

\section{Erst-| Aufnahmebefund}

Die Patientin zeigte einen roten Dermographismus bei unauffälligem Integument.

\section{Befunde diagnostischer Untersuchungen}

Das Gesamt-IgE lag mit $90 \mathrm{kU} / \mathrm{l}$ ebenso wie die Tryptase mit $5,9 \mu \mathrm{g} / \mathrm{l} \mathrm{im} \mathrm{Normbereich.} \mathrm{Das} \mathrm{spezifische} \mathrm{IgE} \mathrm{im} \mathrm{Serum} \mathrm{war} \mathrm{ge-}$ genüber Soja mit 0,53 kU/l (CAP-Klasse 1) und Dermatophagoides farinae mit $1,13 \mathrm{kU} / 1$ erhöht (CAP-Klasse 2), während es gegenüber den deklarierten Inhaltsstoffen der verzehrten Rumkugel (Gummi arabicum, Milcheiweiß, Kakao, Weizenmehl) keine Auffälligkeiten zeigte. Ebenso normwertig waren das spezifische IgE gegenüber Gewürz-, Gräser-, Schimmel- und Kräutermischung sowie gegenüber Erdnuss, Haselnuss, Mandel, Hühnereiweiß, Guarkern, Johannisbrot, Kaffee, alpha-Amylase und dem rekombinanten Gly $m 4$ des Sojas. Im Prick-Test auf ubiquitäre Aeroallergene, Vorratsmilben und einzelne Nahrungsmittel zeigte sich lediglich eine schwach positive Reaktion auf Soja.

\section{Ergänzende Untersuchungen}

Wir nahmen die Patientin wegen der letztendlich ungeklärten Reaktion zur Nahrungsmittelprovokation in Notfallbereitschaft und unter Infusionsschutz stationär auf. Sojamilch (Alpro-Soja ${ }^{\circledR}$ ) sowie Rumkugeln eines anderen Herstellers wurden von der Patientin problemlos vertragen. Bei der Provokation mit der verdächtigen Rumkugel reagierte sie bereits beim Kauen (Provokationsstufe 2) mit Kratzen im Hals, Globusgefühl, beginnender Dyspnoe und einer hypertensiven Entgleisung. Die Provokation wurde daraufhin abgebrochen. Durch die positive Reaktion auf die verdächtige Rumkugel, bei Ausschluss von Sensibilisierungen auf die deklarierten Bestandteile, musste von einer Sensibilisierung gegen eine nicht-deklarierte Zutat ausgegangen werden. Auf gezielte Nachfrage beim Hersteller wurde als weiterer Inhaltsstoff Lupinenmehl angegeben. Das für unsere weiteren Testungen verwandte Lupinenmehl bezogen wir von einer Ölmühle im süddeutschen Raum, die uns aufgrund der Eigenproduktion eine Reinheit des Mehls zusichern konnte. In der gezielten Diagnostik zeigte sich im Prick-Test eine dreifach positive Reaktion auf Lupinenmehl und ein deutlich erhöhtes spezifisches IgE im Serum gegen Lupinensamen mit 23,5 kU/1 (CAPKlasse 4). Bei der anschließenden oralen Provokation mit Lupinenmehl reagierte die Patientin schon nach der ersten Provokationsstufe (Lippenbenetzung) mit oropharyngealen Symptomen und generalisierter Urtikaria.

\section{Immunoblot-Inhibition}

Zur weiteren Diagnosesicherung und um einen möglicherweise bestehenden Zusammenhang im Sinne einer Kreuzreaktion von Lupine und Soja nachzuweisen, wurde eine Immunoblot-Inhibition durchgeführt. Hierfür wurde lyophilisiertes Lupinenmehlextrakt (Extraktherstellung freundlicherweise von Allergopharma, Reinbek, Deutschland) in PBS (phosphate-buffered-saline) gelöst und die Gesamtprotein-Konzentration mittels BCA-Assay (BCA protein assay, Pierce, Rockford, USA) laut Hersteller-Anweisung bestimmt. Zur Herstellung eines Sojabohnenmehlextraktes wurde Sojabohnenmehl (Sigma-Aldrich, Taufkirchen, Deutschland) in PBS gelöst und für 20 Minuten auf Eis inkubiert. Danach wurden die nicht-löslichen Bestandteile des Mehls für 5 Minu-

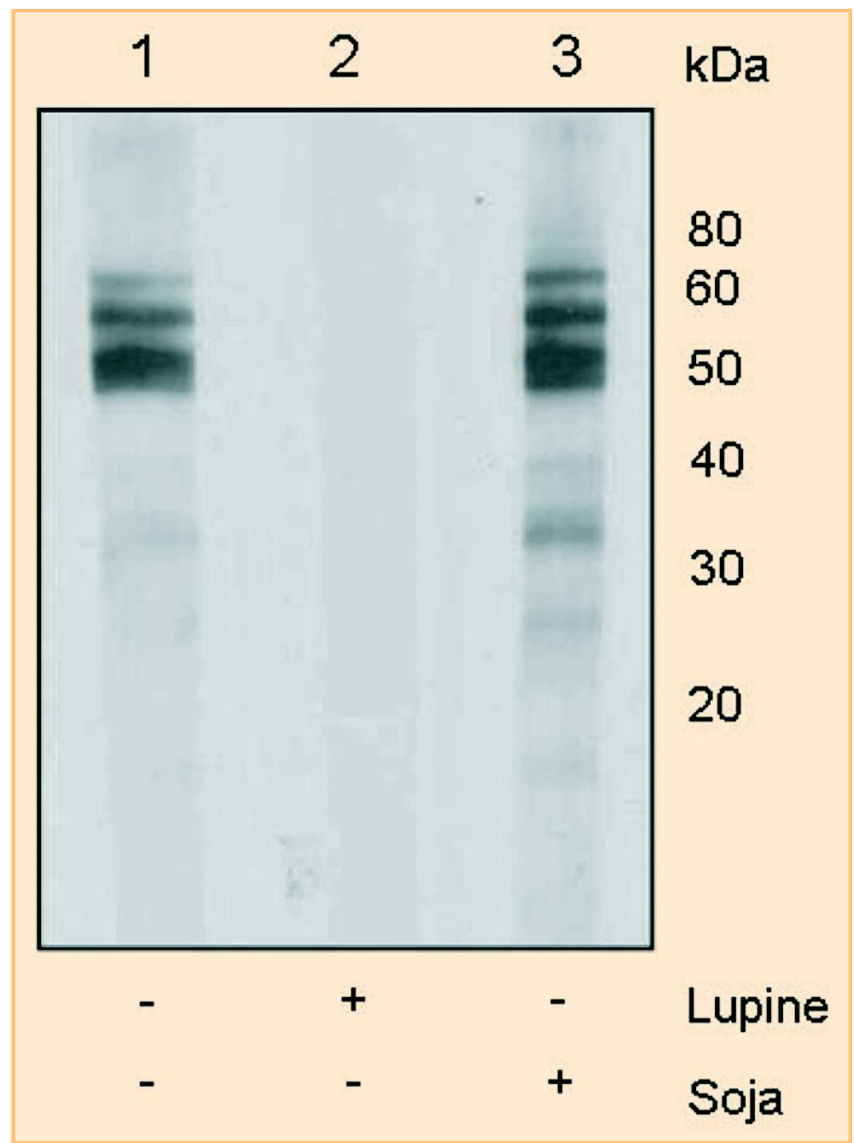

Abb. 1 Immunoblot-Inhibition mit Lupinenmehlextrakt. ImmunoblotStrips mit Lupinenmehlextrakt wurden über Nacht mit dem vorbehandelten Serum der Patientin inkubiert. Für die Inhibitionen wurde Patientenserum 1:3 mit TBS verdünnt und entweder (1) ohne Zusatz, mit (2) $500 \mu \mathrm{g}$ Lupinen- oder (3) $500 \mu \mathrm{g}$ Sojabohnenmehlextrakt über Nacht bei $4{ }^{\circ} \mathrm{C}$ vorinkubiert.

ten bei $13000 \mathrm{rpm}$ (rounds per minute) abzentrifugiert und mit dem Überstand (=Sojabohnenmehlextrakt) weitergearbeitet. Die Gesamtprotein-Konzentration wurde auch hier mithilfe des BCA-Assays ermittelt.

Für die Inhibitionen wurde das Patientenserum 1:3 mit TBS (tris-buffered-saline; $50 \mathrm{mM}$ Tris, $150 \mathrm{mM} \mathrm{NaCl}, \mathrm{pH} 8$ ) verdünnt und mit $500 \mu \mathrm{g}$ Lupinenmehlextrakt oder mit $500 \mu \mathrm{g}$ Sojabohnenmehlextrakt über Nacht bei $4{ }^{\circ} \mathrm{C}$ vorinkubiert. Als Kontrolle diente verdünntes Patientenserum ohne Zusatz. Am Folgetag wurde eine SDS-PAGE (Sodiumdodecylphosphat-Polyacrylamidgelelektrophorese) nach Laemmli [1] durchgeführt. Es wurden $250 \mu$ g Lupinenmehlprotein auf ein $15 \%$ iges SDS-Polyacrylamidgel aufgetragen und nach dem Gel-Lauf auf eine Polyvinylidendifluorid-Membran (PVDF, Immobilon-P Transfer Membran, Millipore, Bedford, MA, USA) transferiert. Diese wurde für 1 Stunde bei Raumtemperatur mit Magermilchpulver blockiert. Nach dreimaligem Waschen mit TBST $(50 \mathrm{mM}$ Tris, $500 \mathrm{mM}$ $\mathrm{NaCl}, 0,05 \%$ Tween 20, pH 8) wurde die Membran über Nacht bei $4{ }^{\circ} \mathrm{C}$ mit dem vorbehandelten Patientenserum inkubiert. Nach erneutem dreimaligem Waschen wurde mithilfe eines Maus-IgG-anti-human-IgE- und eines Peroxidase-markierten anti-Maus-IgG-Antikörpers das spezifische IgE, welches an die immobilisierten Lupinenmehlproteine binden konnte, detektiert. Das Ergebnis der Immunoblot-Inhibition ist in $\mathbf{A}$ Abb. 1 dargestellt. 
Der Immunoblot mit Lupinenmehlextrakt ( $\bullet$ Abb. 1) zeigt, dass die Sensibilisierung der Patientin gegen Lupine hauptsächlich gegen die höhermolekularen Proteine gerichtet ist. Die Inhibition mit $500 \mu \mathrm{g}$ Lupinenmehlextrakt (Strip 2) war vollständig, eine Vorinkubation des Serums mit $500 \mu$ g Sojabohnenmehlextrakt zeigte keinerlei Inhibition der spezifischen IgE-Antikörper gegen Lupinenmehl (Strip 3). Da bei der Kreuzinhibition (Vorinkubation des Serums mit Sojabohnenmehlextrakt, Lupinenmehlextrakt auf der Membran) keine Reduktion des Bandenmusters auftrat, gehen wir davon aus, dass bei dieser Patientin die Lupinensensibilisierung nicht aus einer primären Sensibilisierung gegen Soja resultiert, sondern dass eine primäre Sensibilisierung gegen Lupine vorliegt.

\section{Deklarationsüberprüfung}

Um die Verbreitung von Lupinenmehl in Backwaren in unserer Region zu prüfen, haben wir alle angebotenen Brotsorten, Waffeln und Brötchengerichte dreier Supermärkte auf die deklarierte Zutat Lupinenmehl oder -schrot überprüft. Es konnte somit festgestellt werden, welche Produkte Süßlupinenbestandteile laut Deklaration definitiv beinhalten. In den 148 untersuchten Lebensmitteln war Lupinenmehl in 93,4\% ( $n=138)$ laut Deklaration nicht enthalten, in 5,3\% $(n=8)$ war mit Lupinenmehl und in $1,3 \%(n=2)$ Lupinenschrot in der Zutatenliste aufgeführt. Hauptsächlich wurden Produkte aus dem Kühlregal wie Baguettes, Hot-Dog-Brötchen, Hamburger-Brötchen und Toastwaffeln als lupinenhaltig deklariert. Allein durch die Auswertung der Zutatenliste konnte allerdings nicht sicher ausgeschlossen werden, dass bei den Produkten, in denen die Lupine nicht als Zutat ausgewiesen ist, undeklarierte Lupinenbestandteile enthalten sind.

\section{Therapie und Verlauf}

Wir haben der Patientin bei nachgewiesener primärer Sensibilisierung auf Lupinenproteine geraten, in Zukunft auf lupinenhaltige Produkte zu verzichten und ein Notfallset bei sich zu tragen.

\section{Diskussion}

$\nabla$

In den letzten Jahren wird Lupinenmehl zunehmend wegen seiner hohen Wasserbindungskapazität und guten Emulgatoreigenschaften als preisgünstiger Zusatz in Fertigprodukten oder Backwaren verwendet. Süßlupinen (Lupinus albus, Lupinus luteus, Lupinus angustofolius) spielen des Weiteren aufgrund günstiger ernährungsphysiologischer Eigenschaften und ihrer sojaähnlichen Eiweißzusammensetzung insbesondere in Speisen für Vegetarier, Milcheiweißallergiker und in der glutenfreien Ernährung eine zunehmende Rolle, wobei die Produkte als besonders allergenarm und für Sojaallergiker geeignet beworben werden.

1994 wurde der erste Fallbericht einer Lupinenallergie veröffentlicht, als ein fünfjähriges italienisches Mädchen mit bekannter Erdnussallergie nach dem Genuss lupinenmehlhaltiger Spaghetti mit Urtikaria und Angioödem reagierte [2]. Diese Publikation war der Beginn einer Serie von Einzelfallberichten. Als häufigstes Bild zeigt sich die Lupinenallergie als oral vermittelte Allergieauslösung bei Erdnussallergikern, allerdings sind orale, inhalative und andere Expositionswege auch bei Nicht-Erdnussallergikern als symptomauslösend beschrieben worden [3-5]. Die genaue Prävalenz ist letztlich unbekannt, sicherlich auch weil Lupinenmehl sogar in Fachkreisen als potenzielles Allergen häufig nicht wahrgenommen wird. Wie groß jedoch die Bedeu- tung der Lupinenallergie heutzutage ist, lässt sich daran ablesen, dass Lupinenmehl in Frankreich seit der Zulassung als Nahrungsmittelzusatz im Jahr 1997 dort an vierter Stelle der Lebensmittel steht, die zu anaphylaktischen Reaktionen geführt haben [6]. Erfreulicherweise kann inzwischen parallel zur wachsenden Bedeutung der Lupinenallergie eine zunehmende Forschungsaktivität auf diesem Gebiet verzeichnet werden.

So zeigte sich, dass die isolierte Sensibilisierung gegenüber Lupinen selten ist. Zumeist ist die Allergie Ausdruck einer Kreuzreaktion bei bereits bestehender Sensibilisierung auf andere Leguminosen. Dabei stellen die Kreuzreaktionen mit Erdnüssen den bedeutendsten Anteil, seltener sind Kreuzreagibilitäten mit Sojabohnen, Linsen, grünen Bohnen oder Erbsen beschrieben. Die Hauptallergene stellen, wie bei anderen Leguminosen auch, die Speicherproteine [7]. Die allergenen Proteine der Lupinen sind weitgehend hitzestabil, die beiden Hauptfraktionen stellen die $\alpha$ - und $\beta$-Conglutine, daneben kommen $\gamma$ - und $\delta$-Conglutine vor [8]. Die Allergene sind allerdings molekular noch unzureichend charakterisiert und erst ein Allergen (Lup an 1) ist seit Kurzem in der Allergen-Nomenklatur der I.U.I.S. (International Union of Immunological Societies) geführt. Als mögliche Ursache der Kreuzreaktion bei Erdnussallergikern kann eine hochsignifikante Sequenzhomologie zwischen dem Erdnussallergen Ara $\mathrm{A} 8$ und dem Lupinenprotein PR-10 festgestellt werden. Ein anderes Protein der Lupinen, der beta-conglycin precursor, zeigt signifikante Sequenzhomologien zum Erdnussallergen Ara $h 1$ [9]. Eigenen Untersuchungen nach könnten Kreuzreaktionen mit Sojaproteinen über signifikante Homologien zwischen dem PR-10 von Lupinus albus und dem Gly m 4 (SAM 22) der Sojabohne erklärt werden. Weiterhin besteht zwischen dem $\beta$-Conglycinin des Sojas eine signifikante Sequenzhomologie mit dem Vicilin und Conglutinen der weißen Lupine [10].

Bei unserer Patientin ist die Diagnose einer Lupinenallergie durch den Nachweis der spezifischen Sensibilisierung im Serum und mittels Prick-Test in Kombination mit einer positiven Provokationstestung auf Lupinenmehl gesichert worden. Mittels Immunoblot-Inhibition konnte des Weiteren gezeigt werden, dass eine primäre Sensibilisierung auf Lupine vorliegt und die Lupinensensibilisierung nicht aus einer primären Sojasensibilisierung resultiert.

Lupinenmehl fiel bis vor Kurzem nicht unter die laut EU-Richtlinie zu kennzeichnenden Zutaten. Dadurch war Lupinenmehl als allergieauslösendes Agens, wie auch bei unserer Patientin, schwer auszumachen. Lupinen wurden inzwischen in die Richtlinie der zu deklarierenden Lebensmittelzutaten aufgenommen [11]. Es besteht jedoch weiterhin das Risiko, unbeabsichtigt lupinenhaltige Produkte zu sich zu nehmen, da lose Ware nicht konsequent deklariert werden muss und herstellungsbedingt Spuren von Lupinenmehl in Produkten enthalten sein können. Sollte sich ein entsprechender klinischer Verdacht einer Lupinenallergie nicht wie in unserem Fall durch Deklarationsüberprüfung oder Herstelleranfrage bestätigen lassen, ist eine Detektion von Lupinenbestandteilen im entsprechenden Nahrungsmittel mittels proteinbiochemischer oder molekularbiologischer Methoden möglich. Die von uns durchgeführte Deklarationsuntersuchung zeigt, dass insbesondere Fertigbackwaren aus dem Kühlregal Lupinenmehl enthalten, welches wegen seiner hohen Wasserbindungskapazität die Lebensmittel frisch hält und vor dem Austrocknen schützt. Mit zunehmender Verwendung von Lupinenprodukten ist auch hierzulande mit einer steigenden Manifestation der Lupinenallergie zu rechnen, die einem im klinischen Alltag begegnen kann. Unser Fall ist ein Beispiel dafür, 
dass bei ungeklärten Reaktionen auf Teig-, Brot- und Backwaren sowie Fertiggerichte eine Lupinenallergie die Ursache sein kann, an die gedacht werden muss. Lupinenprodukte sollten, besonders im Zusammenhang mit den immer häufiger auftretenden Fallberichten, nicht mehr unkritisch als weniger allergene Sojaalternative angesehen werden. Häufig können insbesondere bei Erdnuss- und Sojaallergikern Kreuzreaktionen gegenüber Lupinen beobachtet werden, aber auch die seltenen Neusensibilisierungen können - wie im Falle unserer Patientin - von ausgesprochener klinischer Relevanz sein.

\section{Abstract}

\section{Lupine Flour - An Allergen Gaining in Importance} $\nabla$

A 48-year-old woman who had complained of oropharyngeal symptoms and dyspnea after ingestion of a rum truffle was referred to our department. Apart from a clinically not relevant sensitisation against soybean, the initial allergological analyses were without any pathological findings. Only due to a positive oral provocation with the rum truffle under inpatient conditions and after enquiring the manufacturer about additional undeclared ingredients, lupine flour was identified and confirmed to be the responsible allergen. Immunoblot-inhibition revealed a primary sensitisation against lupine flour. Lupine flour has increasing importance as a food ingredient especially in bakery products. Our case report aims to sensitize the readership to this allergen and advance early diagnosis of this increasing important allergy.

\section{Literatur}

1 Laemmli UK. Cleavage of structural proteins during the assembly of the head of bacteriophage T4. Nature 1970; 227: 680-685

2 Hefle SL, Lemanske RF Jr., Bush RK. Adverse reaction to lupine-fortified pasta. J Allergy Clin Immunol 1994; 94: 167 - 172

3 Parisot L, Aparicio C, Moneret-Vautrin DA, Guerin L. Allergy to lupine flour. Allergy 2001; 56: $918-919$

4 Matheu V, de Barrio M, Sierra Z, Gracia-Bara MT, Tornero P, Baeza ML. Lupine-induced anaphylaxis. Ann Allergy Asthma Immunol 1999; 83: $406-408$

5 Novembre E, Moriondo M, Bernardini R, Azzari C, Rossi ME, Vierucci A. Lupin allergy in a child. J Allergy Clin Immunol 1999; 103: 1214-1216

6 Moneret-Vautrin DA, Kanny G, Morisset M, Rance F, Fardeau MF, Beaudouin $E$. Severe food anaphylaxis: 107 cases registered in 2002 by the allergy vigilance network. Eur Ann Allergy Clin Immunol 2004; 36: $46-51$

7 Leduc V, Moneret-Vautrin DA, Guerin L. Allergenicity of lupin flour. Allerg Immunol (Paris) 2002; 34: 213 - 217

8 Foss N, Duranti M, Magni C, Frokiaer H. Assessment of lupin allergenicity in the cholera toxin model: Induction of IgE response depends on the intrinsic properties of the conglutins and matrix effects. Int Arch Allergy Immunol 2006; 141: 141 - 150

9 Guarneri F, Guarneri C, Benvenga S. Identification of potentially crossreactive peanut-lupine proteins by computer-assisted search for amino acid sequence homology. Int Arch Allergy Immunol 2005; 138: $273-277$

10 Grundmann SA, Hemfort P, Luger TA, Brehler R. Computergestützte Aminosäurensequenzanalyse: Können Kreuzreaktivitäten vorhergesagt werden? JDDG 2007; 11: 1076-999

11 Vieths S, Holzhauser T, Erdmann S, Fuchs T, Henzgen M, Kleine-Tebbe J, Lepp U, Niggemann B, Raithel M, Reese I, Saloga J, Vieluf I, Zuberbier T, Werfel $T$. Neue Deklarationspflicht für Nahrungsmittelallergene in Lebensmitteln. Allergologie 2006; 29: $403-409$ 